\title{
El tiempo de tránsito del pulso: un índice de cambios en la presión arterial
}

\section{Enrique G. Fernández-Abascal*}

\section{Universidad Complutense de Madrid}

Las necesidades metodológicas para una mayor comprensión de la psicofisiología cardiovascular, por un lado, y los recientes planteamientos de la medicina comportalmental sobre este área, por otro, exigen de un sistema de medida continuo y no invasivo de la presión arterial (Fernández-Abascal, $1983 \mathrm{a}$ ).

El tiempo de tránsito del pulso (TTP) parece ser una solución para la medida latido a latido de cambios en la presión arterial. En un trabajo anterior (Fernández-Abascal, 1983 b) se estableció que la mejor forma de detección del TTP, para su utilización como índice de la presión arterial, define a éste como el intervalo de tiempo que transcurre desde la cresta de la onda $R$ del electrocardiograma (EKG) hasta la llegada del pulso a la arteria radial (ver figura $\mathrm{I}$ ).

\section{Figura I}

Medida del tiempo de tránsito del pulso (TTP), entre la onda $\mathrm{R}$ del electrocardiograma (EKG) y la subida sistólica del pulso radial (PR).

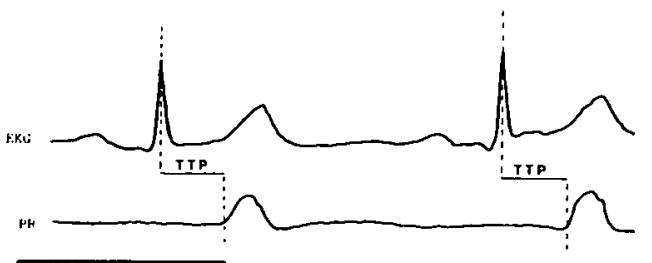

El propósito de este trabajo se centró, en primer lugar, en validar el TTP como indice de la presión arterial y en estudiar la influencia del intervalo de tiempo intracardiaco que se suma al verdadero TTP, cuando éste se estima como hemos definido anteriormente. Así pues, la comprobación de en qué medida el TTP sigue a la presión arterial a lo largo de un amplio rango de éstas y en qué medida el segmento del período de preeyección (PPE) incluido en el TTP es constante o covaría con la presión arterial, o, por el contrario, su contribución distorsiona la relación entre el TTP y la presión arterial, son las dos primeras finalidades planteadas.

Aunque muy escasos, los trabajos existentes parecen apuntar a que la citada contribución del PPE no es constante. Obrist, Light, McCubbin, Hutcheson y Hoffer (1979) encontraron que el bloqueo beta-adrenérgico alteró las correlaciones entre el TTP y la presión sistólica, debiéndose esta atenuación a la influencia que el bloqueo ejerció sobre el TTP, pero no sobre la presión sistólica; así pues, decrementos en el T'TP pueden reflejar influencias beta-adrenérgicas por acortamiento del PPE, mientras que la presión arterial es minimamente influenciada. Aunque este hecho no parece afectar a todas las

* Dirección del autor: Dpto. Psicología General, Facultad de Psicología. Campus de Somosaguas. 28023 Madrid. 
condiciones, así Newlin y Levenson (1979), mediante entrenamiento en feedback de TTP, encontraron que los cambios significativos logrados en el TTP eran de similar magnitud a los obtenidos en el verdadero TTP, no encontrándose efectos significativos sobre el PPE.

Una tercera finalidad de este trabajo fue la de someter a prueba un sistema para la estimación de la reactividad en la presión arterial, reactividad cuya importancia ha sido puesta de relieve por Obrist (1981), Obrist, Light, Hastrup y Langer (1981) o Steptoe (1982), como precursora de la hipertensión esencial. Los intentos de evaluación basados en la estimación de la reactividad frente a una tarea estresante, contrastada con una línea base pretarea, han proporcionado resultados válidos en algunas ocasiones (Hines y Brown, 1936; Shapiro, 1961; Shapiro, Moustsos y Krifcher, 1963), pero en otras fallaron en su intento (Boyer, Fraser y Doyler, 1960; Remington, Lambrath, Moser y Hobler, 1960; Harlan, Osborne y Graybill, 1964). Recientemente (Light y Obrist, 1980a, 1980b; Obrist, I98 I; Manuck y Proietti, 1982) se ha encontrado que la mejor forma de evaluación de la reactividad se logra contrastando la ejecución en una tarea estresante con una línea base de relajación, en la que las influencias comportamentales estén minimizadas. La comprobación de si la variabilidad en el TTP, estimada mediante una tarea de aritmética mental y una línea base de relajación, da razón de la variabilidad de la presión sistólica a lo largo de todas las tareas experimentales, es la tercera finalidad de este trabajo.

Por último, se intentó estudiar el efecto de caída en la actividad de la respuesta cardiovascular a lo largo de la sesión experimental, efecto sistematizado teóricamente por Gavalas (1967), el cual representa un serio problema en los trabajos de feedback de presión arterial, puesto que confunde los resultados del entrenamiento cuando el control es medido únicamente como cambios desde el nivel pretratamiento (Shapiro, Tursky y Swartz,
1970; Fey y Lindholm, 1975). Asimismo, se intentó probar la bondad del sistema de control propuesto por Steptoe (1976), utilizando un doble análisis en relación a la línea base preexperimental y pretarea.

Las hipótesis que se someten a prueba en este trabajo son:

I. El TTP está relacionado de forma inversa con la presión sistólica.

2. La porción del PPE cardíaco incluido en el TTP varía, independientemente de éste, de unas tareas experimentales a otras.

3. La diferencia individual en TTP, entre una tarea de aritmética mental y una línea base de relajación, será un buen predictor de reactividad en presión sistólica.

4. Los sujetos en situación de inmovilización sufrirán una caída en sus presiones arteriales a lo largo del tiempo.

\section{METODO}

\section{Sujetos}

Los sujetos que participaron en este trabajo fueron 24 estudiantes voluntarios, de los cuales 16 eran hombres y 8 mujeres, con un rango de edades entre los 21 y los 34 años $(\bar{x}=23,3)$; sus presiones arteriales previas al experimento se encontraban dentro del rango normotensivo y no se les conocía ningún trastorno cardiovascular, ni se encontraban tomando ninguna medicación a tal respecto en el momento del examen.

Todos los sujetos fueron informados, previamente a obtener su conformidad en la participación, de que la finalidad del experimento era la recogida de algunas variables fisiológicas tales como la presión arterial, el pulso arterial y el EKG; y asimismo, que el experimento no requería de ninguna actividad especial por su parte. 
Tres sujetos tuvieron que ser eliminados y sustituidos por otros tantos, para completar el número final de 24, por dificultades de procedimiento.

\section{Material}

El EKG fue tomado desde la segunda derivación bipolar de las extremidades, procesado por un amplificador de EKG y registrado sobre un polígrafo de cuatro canales (Lafayatte Instrument Co., modelo $76 \mathrm{r}_{02}$ ). El pulso de la arteria radial fue detectado en la muñeca del brazo derecho, a través de un sensor piezoeléctrico y registrado sobre un segundo canal del citado polígrafo. La velocidad de arrastre del papel fue de $30 \mathrm{~mm} / \mathrm{seg}$.

La presión arterial fue recogida esfigmomanométricamente, a través de un manguito de presión colocado alrededor del brazo izquierdo del sujeto. Los sonidos de Korotkoff fueron detectados por un micrófono de cristal situado entre el manguito y la arteria braquial. Ambas señales, presión y sonidos de Korotkoff, fueron procesados por un esfigmomanómetro semiautomático (Nihon Seimitsu Sokki Co., modelo DS-IO2), el cual determinó y presentó digitalmente las presiones sistólica (definida como la presión existente en el manguito en el momento de producirse el primer sonido de Korotkoff), diastólica (definida como la presión existente en el manguito en el momento de producirse al último sonido) y la tasa de deflacción de la presión del manguito, la cual se mantuvo constante durante el tiempo de medida a $2 \mathrm{mmHg} / \mathrm{seg}$. El manguito fue inflado manualmente a una presión superior a $30 \mathrm{mmHg}$. a las presiones sistólicas registradas previamente, $\mathrm{y}$ en las primeras lecturas de las tareas experimentales el inflado se aumentó en $40 \mathrm{mmHg}$. sobre las lecturas del periodo de descanso previo. Los sonidos de Korotkoff fueron registrados sobre el trazado poligráfico junto con las señales anteriormente indicadas y la respiración del sujeto, mientras que las presiones sistólicas y diastólicas se registraron manualmente.
Las condiciones de luz y temperatura de la cabina experimental fueron mantenidas constantes a lo largo de toda la sesión y de todos los sujetos. La citada cabina contenía, además de los instrumentos de medida y registro descritos anteriormente, una mesa exploratoria donde el sujeto permaneció recostado; esta mesa estaba situada de tal modo que el sujeto no tuvo acceso visual a los registros en ningún momento de la sesión experimental.

\section{Procedimiento}

La sesión experimental se realizó con los sujetos recostados en la posición de decúbito dorsal, y las tareas de que constó el procedimiento fueron las siguientes:

A) Linea base.-En primer lugar se registró una línea base con la única indicación a los sujetos de que permanecieran recostados. A lo largo de diez minutos se realizaron mediciones continuas de los registros poligráficos y io ciclos de medida de la presión arterial.

B) Maniobra de Valsalva.--Esta maniobra consiste en expiraciones forzadas junto con el cierre del glotis, produciendo un marcado aumento de la presión intratorácica; sus efectos sobre el lado izquierdo del corazón y sobre el sistema arterial son, de modo inmediato, un ligero aumento de llenado del ventrículo izquierdo con expresión del reservorio pulmonar, un ligero aumento del gasto sistólico del ventrículo izquierdo y un ligero aumento de la amplitud del pulso con bradicardia refleja eventual e incremento del tono simpático. El factor más importante es la transmisión de la presión intratorácica elevada al sistema aórtico, con la consiguiente elevación de las presiones sistólica y diastólica. Esta maniobra fue elegida porque la elevación de las presiones se produce sin causar alteraciones en el PPE (Flessas, Kumar y Spodick, 1970). Durante la ejecución de esta maniobra se realizaron seis lecturas de la presión arterial, además de los consiguientes registros de las medidas continuas. 
C) Periodo de descanso.-Un período de descanso de cuatro minutos de duración, en el cual se realizaron otras tantas mediciones de la presión, y que precedió a la siguiente tarea experimental sirviendo de línea base pretarea.

D) Aritmética mental.-Se instruyó a los sujetos para que contaran de forma inversa y lo más rápidamente posible desde cien hasta cero (de uno en uno) y desde setecientos hasta cero (de tres en tres). Estas dos tareas le fueron presentadas al sujeto a lo largo de seis minutos, mientras se realizaron otras seis de la presión arterial. Este procedimiento se ha comprobado que produce una elevación en las presiones sistólicas y diastólicas, junto con un incremento en la tasa cardíaca (Steptoe, Smulyan y Gribbin, 1976; Allen, Schneider, Davidson, Winchester y Taylor, 1981).

E) Periodo de descanso.-Un nuevo período de descanso pretarea de cuatro minutos, con sus consiguientes mediciones, precedió a la tarea siguiente.

F) Contracción muscular isométrica.-Los sujetos fueron intruidos a presionar su mano izquierda tan fuerte como pudieran contra la mesa exploratoria, produciendo contracciones isométricas. Esta tarea se repitió a lo largo de seis minutos, mientras se realizaron seis nuevas lecturas de la presión arterial. Esta prueba, además de aumentar la tasa cardíaca, acelera el retorno venoso y aumenta el gasto cardíaco, produciendo elevaciones en la presión que cabe esperar se reflejen distorsionadamente en el TTP, debido a las influencias de esta maniobra sobre la dilatación intracardíaca (Quarry y Spodick, I 974).

G) Periodo de descanso.-Período de separación entre las tareas experimentales igual que los anteriores.

H) Instrucciones de relajación.-La última tarea consistió en unas breves instrucciones de relajación, que el sujeto recibió por un período de diez minutos, y que se centraron principalmente sobre la laxitud muscular y la cadencia respiratoria. A lo largo de esta tarea se realizaron diez mediciones de la presión arterial. El resultado esperado es una disminución tanto de la presión como de la tasa cardíaca del sujeto.

Todas las sesiones se comenzaron con la linea base y terminaron con las instrucciones de relajación; sin embargo, la presentación de las otras tres tareas (maniobra de Valsalva, aritmética mental y contracción isométrica) se presentaron de forma contrabalanceada, de tal modo que a cuatro sujetos se les aplicó el orden A-B-C-D-E-F-G-H; a otros cuatro, el A-D-C-B-E-F-G-H; a otros, A-F-C-D-EB-G-H, hasta completar con cuatro sujetos cada una de las seis posibles combinaciones cronológicas de estas tres tareas, que estuvieron siempre separadas entre ellas por un período de descanso, que conjuntamente con los últimos cuatro minutos de la línea base inicial sirvieron como línea base pretarea continuada a lo largo de toda la sesión experimental.

\section{Análisis de datos}

Los datos sometidos a análisis fueron las cincuenta lecturas de las presiones sistólica y diastólica, así como de la presión arterial media calculada como la presión diastólica menos un tercio de la presión del pulso. El TTP se obtuvo por cálculo en milisegundos (mseg.) del tiempo transcurrido entre la cresta de la onda $\mathrm{R}$ del EKG y el punto de inflexión de la subida sistólica del pulso radial; el cálculo se hizo a partir de los registros poligráficos con una resolución de $3,33 \mathrm{mseg}$., mediante una doble valoración realizada independientemente por dos correctores. La actividad cardíaca fue calculada como el intervalo entre latidos (IEL), expresado en mseg. el tiempo entre dos ondas $R$ consecutivas. Las mediciones del TTP y el IEL fueron realizadas para las detecciones primera y última de los sonidos de Korotkoff de cada ciclo de medida, utilizándose tanto las mediciones de los citados ciclos cardíacos como de los cinco anteriores y cuatro posteriores. 


\section{RESULTADOS}

\section{Relación entre variables}

Las variables fisiológicas fueron correlacionadas entre sí para cada uno de los
24 sujetos que componían la muestra. Los resultados de este análisis se recogen en la tabla $\mathrm{I}$, donde se presentan para cada sujeto las correlaciones del TTP con las presiones arteriales y el IEL, así como de éstas entre sí.

\section{TABLA I}

Coeficientes de correlación para cada sujeto, entre las variables: tiempo de tránsito del pulso (TTP), intervalo entre latidos (IEL) y las presiones sistólica (PS), diastólica (PD) y media (PAM).

\begin{tabular}{|c|c|c|c|c|c|c|c|}
\hline TTP-PS & ITP-PD & TTP-PAM & TIP-IEL & PS-IEL & PD-IEL & PAM-IEL & PS-PD \\
\hline$-.804 * * *$ & .065 & $-.355^{*}$ & $.445^{* *}$ & $-.425 * *$ & .185 & -.176 & $.336^{*}$ \\
\hline$-.880^{* * *}$ & -.273 & $-.491 * * *$ & .249 & -.273 & -.239 & -.188 & $.397^{* *}$ \\
\hline$-.613^{* * *}$ & .016 & $-.286^{*}$ & .244 & -.263 & .102 & -.034 & .255 \\
\hline$-.650 * * *$ & $-.544 * * *$ & $-.676^{* * *}$ & $.313^{*}$ & $-.383^{*}$ & -.016 & -.269 & $.441^{* *}$ \\
\hline$-.844 * * *$ & $-.311^{*}$ & $-.703^{* * *}$ & $.322^{*}$ & -.354 & -.085 & $-.286^{*}$ & $.280^{*}$ \\
\hline$-.685^{* * *}$ & $-.443^{* *}$ & $-.722 * * *$ & .061 & -.230 & -.238 & -.221 & .224 \\
\hline$-.867 * * *$ & -.133 & $-.608^{* * *}$ & .176 & -.192 & .069 & -.090 & .163 \\
\hline$-.964 * * *$ & $-.369 * *$ & $-.742 * * *$ & .043 & .046 & -.055 & -.106 & $.536 * * *$ \\
\hline$-.963 * * *$ & $-.451 * *$ & $-.896^{* * *}$ & .276 & $-.448^{* *}$ & $-.337^{*}$ & $-.452^{* * *}$ & $.601 * * *$ \\
\hline$-.821 * * *$ & -.267 & $-.785 * * *$ & $.381 *$ & $-.311 *$ & -.091 & $-.366^{* *}$ & .181 \\
\hline$-.796 * * *$ & $-.310^{*}$ & $-.548 * * *$ & .153 & -.126 & .194 & .010 & $.348 *$ \\
\hline$-.878 * * *$ & .149 & -.159 & $.340^{*}$ & $-.459 * * *$ & $.438^{* *}$ & $.281 *$ & -.172 \\
\hline$-.926 * * *$ & -.161 & $-.581 * * *$ & $.297 *$ & -.020 & -.011 & -.216 & -.047 \\
\hline$-.949 * * *$ & $-.513 * * *$ & $-.761 * *$ & $.449 * *$ & $-.435 * *$ & $-.472^{* * *}$ & $-.402^{* *}$ & $.374 * *$ \\
\hline$-.893^{* * *}$ & $-.332^{*}$ & $-.631 * * *$ & .117 & -.250 & $-.364 * *$ & -.218 & $.305^{*}$ \\
\hline$-.820 * * *$ & $-.500 * *$ & $-.873^{* * *}$ & .158 & -.072 & -.091 & -.144 & $.302^{*}$ \\
\hline$-.982 * * *$ & .278 & -.249 & .227 & -.159 & .162 & .045 & -.076 \\
\hline$-.986 * * *$ & $-.307^{*}$ & $-.502 * * *$ & .083 & $-.314 *$ & -.107 & $-.361 * *$ & .172 \\
\hline$-.933^{* * *}$ & -.278 & $-: 455^{* * *}$ & $.340^{*}$ & $-.420^{* *}$ & -.088 & -.185 & .276 \\
\hline$-.988 * * *$ & .003 & $-.566^{* * *}$ & .264 & -.216 & -.215 & -.204 & .144 \\
\hline$-.720^{* * *}$ & $-.489 * * *$ & $-.728^{* * *}$ & $.490 * * *$ & $-.474 * * *$ & $-.620^{* * *}$ & $-.532^{* * *}$ & $.645 * *$ \\
\hline$-.997^{* * *}$ & $-.558^{* * *}$ & $-.723^{* * *}$ & $.335^{*}$ & $-.313^{*}$ & -.226 & -.126 & $.412^{*}$ \\
\hline$-.855^{* * *}$ & -.222 & $-.810^{* * *}$ & -.006 & -.137 & $.515 * * *$ & -.024 & .162 \\
\hline$-.958 * * *$ & $-.575^{* * *}$ & $-.609 * * *$ & .222 & $-.333^{*}$ & $-.453^{* * *}$ & -.261 & $.456 * * *$ \\
\hline
\end{tabular}

$\mathrm{N}=50 ; * \mathrm{p}<.05 ; * * \mathrm{p}<.01 ; * * \mathrm{p}<.001$

El TTP covarió negativamente con las presiones arteriales media, diastólica y sistólica, siendo con esta última con quien obtuvo las correlaciones más altas y significativas para todos los sujetos (rango de -.613 a -.997 ); la correlación media entre el TTP y la presión sistólica fue de $-.866, \mathrm{p}<.001$. Las correlaciones entre el TTP y la presión arterial media fueron también altas y negativas, aunque con valores más atenuados que en el caso anterior (rango de -.159 a -.896 ), con una correlación media de $-.602, \mathrm{p}<.001$; mientras que las correlaciones del TTP con la presión diastólica, aunque casi en su mayoria de signo negativo, raramente 
alcanzaron la significación estadistica (rango de .278 a -.575 ), con una correlación media de $-.273, \mathrm{P}>.05$.

Las correlaciones del IEL con el TTP y con las presiones arteriales fueron todas bajas, llegando en ocasiones a superar el nivel de significación. En el caso de la correlación entre el IEL y el TTP (rango de -.006 a .490) la correlación media fue de .249, P > .os; siendo la correlación del IEL con la presión sistólica muy semejante a la obtenida con el TTP, pero de signo contrario dada su relación inversa (rango de $.046 \mathrm{a}-.474$ ), con una correlación media de $-.273, \mathrm{p}>$.05. Las correlaciones del IEL tanto con la presión diastólica (rango de .5 is a - .620) como con la presión media (rango de $.28 \mathrm{I}$ a -.532 ), fueron también mayoritariamente negativas pero más inconsistentes, obteniéndose unos valores medios de, repectivamente, $-.085, \mathrm{p}>.05, \mathrm{y}-.188$, $\mathrm{p}>$.05. Por tanto, dada la relación inversa entre el IEL y la tasa cardiaca, la relación de ésta con la presión arterial es positiva, pero muy baja. Ias correlaciones entre las presiones sistólica y diastólica fueron moderadas y mayoritariamente positivas, aunque también bastante dispersas (rango de -.172 a .645 ), con un valor medio de .280, $\mathrm{p}<.05$.

Tal y como se planteaba en la primera hipótesis, la relación entre el TTP y la presión sistólica es inversa y uniformemente alta en todos los sujetos; además, se presuponía que esta relación era mayor con la presión sistólica que con las restantes presiones; así, la relación del TTP con la presión sistólica se mostró significativamente mayor que con la presión diastólica $[\mathrm{t}(23)=1.936, \mathrm{p}<.05]$ y que con la presión media $[t(23)=1.816, p<$. os $]$. Por otra parte, se encontró que las covariaciones del IEL con el TTP y con la presión sistólica no difirieron significativamente $[t(23)=0.736, p>05]$, por lo que cabe suponer que el TTP es un buen indice de cambios en la presión sistólica. Adicionalmente, se calcularon las líneas de regresión del TTP sobre la presión sistólica, para ser utilizadas en un análisis posterior, comprobándose la bondad del ajuste li- neal de esta relación; en la figura 2 se muestra la representación gráfica para el caso de los dos sujetos que obtuvieron las mejores correlaciones y de los que obtuvieron las peores.

\section{Efecto de las manipulaciones}

Se realizó un análisis preliminar de las tareas experimentales por medio de análisis de varianza de medidas repetidas para cada una de las variables fisiológicas: TTP, IEL y presiones arteriales (en este primer análisis, los datos fueron tratados con referencia a la línea base inicial).

Las manipulaciones de las tareas experimentales produjeron efectos significativos tanto sobre el TTP $[F(6 / 138)=14.69$, $\left.\mathrm{MC}_{\mathrm{e}}=49.9^{8}, \mathrm{p}<.001\right]$, como sobre las presiones sistólica $[F(6 / 138)=18.32$, $\mathrm{MC}_{\mathrm{e}}=14.25, \mathrm{p}<.001 \mathrm{I}$, diastólica $\left[F(6 / 138)=4.98, M C_{c}=14.26, p<.001\right] y$ media $\left[F(6 / 138)=11.06, \quad M_{e}=8.93\right.$, $\mathrm{p}<.00 \mathrm{r}]$. Los efectos de las tareas sobre todas estas variables fueron muy semejantes; así, por ejemplo, la aritmética mental, que produjo las mayores elevaciones en todas las variables, alcanzó diferencias estadísticamente significativas con relación al resto de las maniobras y períodos de descanso en todas las variables, excepto con las contracciones musculares isométricas en el caso de la presión sistólica y diastólica, ya que en el TTP y en la presión media también se mostró superior a ésta. Las instrucciones de relajación también mostraron diferencias significativas con las contracciones musculares isométricas en los casos de las presiones media y sistólica y TTP; y en el caso de estas dos últimas, la relajación, a su vez, también obtuvo los niveles de activación menores que los producidos por la maniobra de Valsalva. El período de descanso que siguió a la maniobra de Valsalva difírió de la tarea precedente así como de las contracciones musculares en el TTP. Y en el caso de la presión sistólica y media, las contracciones musculares difirieron de las restantes tareas, excepto en el caso de la presión sistólica, que, como 
Trazado gráfico del TTP (en mseg.) y la presión sistólica (en $m m \mathrm{Hg}$.) para los dos sujetos que obtuvieron las mejores correlaciones entre ambas variables (parte superior) y la de los sujetos con los valores correlacionales más bajos (parte inferior).
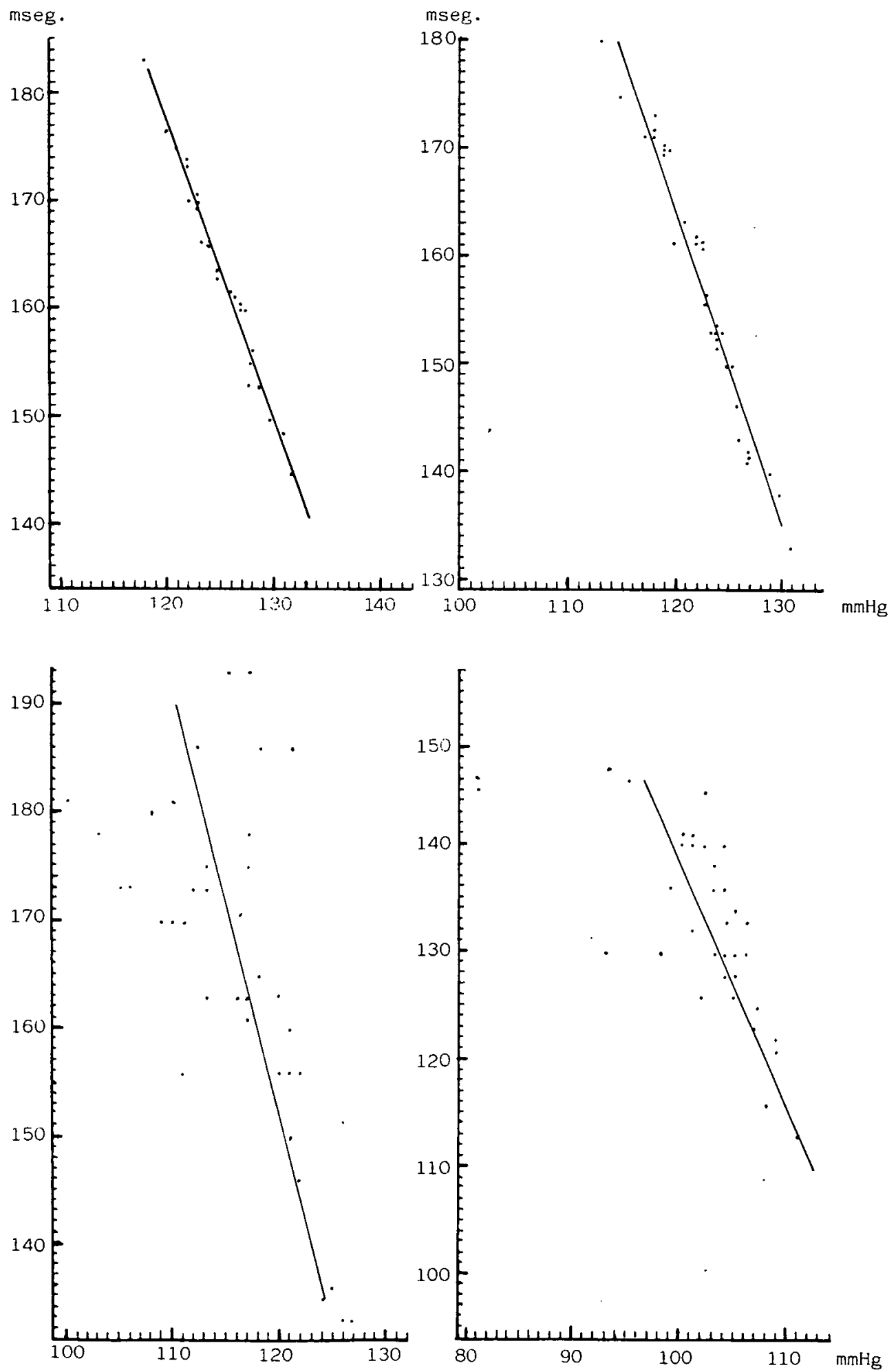

Estudios de Psicologia $10^{\circ} 21 \cdot 1985$ 
ya se indicó, la aritmética mental y las contracciones musculares no mostraron diferencias significativas entre ellas. Asi pues, los mayores niveles de presión arterial, que consiguientemente estuvieron reflejados en el TTP, se obtuvieron a través de la aritmética mental, seguida por las contracciones musculares isométricas, y los niveles más bajos los obtuvo las instrucciones de relajación; mientras que la maniobra de Valsalva obtuvo unos valores intermedios sin significaciones generalizadas, como en el caso de las maniobras anteriores.

El análisis de varianza de las tareas para el caso del IEL produjo también efectos significativos $[\mathrm{F}(6 / 138)=11.66$, $\mathrm{MC}_{\mathrm{e}}=2383.7 \mathrm{I}, \mathrm{p}<.00 \mathrm{I}$, como en el caso de los análisis anteriores la aritmética mental alcanzó diferencias significativas con las restantes maniobras y periodos de descanso, excepto con la maniobra de Valsalva, que, aunque también produjo una alta reducción del IEL, ésta fue menor que la lograda por la aritmética mental. La maniobra de Valsalva, a su vez, alcanzó diferencias significativas con las restantes tareas excepto con la de las contracciones musculares. Las instrucciones de relajación, aunque presentó los IEL más dilatados, sólo obtuvo diferencias significativas con las tres maniobras experimentales, pero no con los periodos de descanso pretarea.

Así pues, traduciendo los resultados anteriores a términos de tasa cardíaca, la relajación fue la tarea que produjo los niveles más bajos de tasa, mientras que la aritmética mental y la maniobra de Valsalva fueron las que produjeron las tasas mayores; las contracciones musculares presentaron unas tasas intermedias con dos únicas diferencias significativas; por arriba difirió con la aritmética mental y por abajo con las instrucciones de relajación.

Para realizar la comprobación de la segunda hipótesis, relativa a si la porción del PPE que contiene el TTP se mantiene constante o varia en la misma magnitud y sentido que el TTP, o por el contrario varia de modo independiente y consiguientemente distorsiona diferencialmente la relación TTP-presión sistólica de unas situaciones a otras. $Y$ dado que a través de la metodologia empleada en este trabajo no es posible conocer los valores del PPE o del verdadero T'TP, el análisis alternativo realizado consistió en el cálculo para cada sujeto de la línea de regresión del TTP sobre la presión sistólica $y$, posteriormente, a partir de los datos del TTP para cada tarea experimental, predecir los valores de presión sistólica esperados en función de la regresión y someter a análisis las puntuaciones diferenciales entre las presiones sistólicas reales, medidas en el experimento, y las presiones sistólicas que resultaron de la predicción a través de las puntuaciones del TTP. De este modo, si la aportación que el TTP recibe del PPE es constante o covaría puntualmente con el verdadero TTP, no deberian encontrarse diferencias significativas entre ninguna de las tareas; por el contrario, si la aportación del PPE es diferente de una situación a otra, habrá que esperar que aparezcan diferencias significativas entre las tareas en función de esa aportación diferencial.

Sometidas a un análisis de varianza de medidas repetidas las puntuaciones diferenciales de cada una de las tareas experimentales, aparecieron diferencias estadísticamente significativas $[\mathrm{F}(7 / 16 \mathrm{I})=2.25$, $\left.\mathrm{MC}_{\mathrm{e}}=6.42, \mathrm{p}<.05\right]$; realizadas las diversas comparaciones de pares de tareas, aparecieron diferencias significativas entre la maniobra de las contracciones musculares isométricas y todos los descansos pretarea. Asi pues, parece que la porción del PPE contenida en el TTP se comporta de modo diferencial de unas situaciones a otras; en nuestro caso, en las situaciones de reposo sin ningún tipo de contractura muscular, su comportamiento ha sido distinto de la situación de contracción muscular.

La comprobación de la tercera hipótesis se realizó correlacionando las diferencias en TTP entre aritmética mental y relajación con las desviaciones típicas de la presión sistólica a lo largo de todas las 


\section{Estudios}

tareas experimentales, obteniéndose una correlación para el total de la muestra de .439, P <.05; además, el 12 por 100 de los sujetos que obtuvieron las mayores diferencias en el TTP coincidieron puntualmente con ser los que presentaron la mayor variabilidad en presión sistólica a lo largo de toda la sesión experimental. La correlación alternativa entre las diferencias de aritmética mental y la línea base inicial con la desviación típica de la presión sistólica, no llegó a alcanzar la significación estadística (.282); asimismo, los niveles preexperimentales de la propia presión sistólica también fracasaron en dar respuesta a su variabilidad experimental $(.032)$. Por tanto, la hipótesis propuesta queda confirmada, ya que la diferencia entre las tareas de aritmética mental y relajación no sólo parece ser un buen indice de reactividad en presión sistólica, sino que es claramente mejor que los que habitualmente son utilizados, como es el caso de las mediciones "casuales" de la presión o la reactividad en una tarea estresante, pero en relación a una línea base pretarea. Adicionalmente se comprobó si esta predicción se cumplía también para el caso de la presión diastólica, no confirmándose este punto, ya que no se obtuvo significación estadística $(-.187)$.

La cuarta hipótesis planteada predecía que en las situaciones de medida con relativa inmovilización del sujeto se produciría una caída en la reactividad, lo cual, a su vez, introduce una fuente de confusión para la interpretación de los resultados, ya que esta caida es una fuente de variación no controlada que interfiere con la fuente de variación experimental. Con el fin de solucionar este problema, como se expuso anteriormente, las soluciones adoptadas comúnmente han consistido en la introducción de líneas base pretarea continuadas a lo largo de toda la sesión experimental y en realizar un doble análisis, por un lado con referencia a la linea base inicial y, por otro, referenciando cada tarea a una línea base previa. Para comprobar si en nuestro trabajo había aparecido o no el efecto de caída $y$, en su caso, comprobar la validez de la citada solución, el diseño se realizó intercalando una línea base pretarea previa a todas las manipulaciones y, por otra parte, las tres maniobras centrales (Valsalva, aritmética mental y contracciones musculares isométricas) fueron presentadas de forma contrabalanceada conformando un diseño factorial de tres maniobras por seis órdenes de presentación; las puntuaciones diferenciales del TTP a las lineas base pretarea fueron sometidas a un análisis de varianza de dos direcciones con una de las medidas repetidas, no apareciendo efectos significativos para el orden de presentación $[F(s / 18)=0.64$, $\left.M C_{c}=1 \pm 1.76, p>.05\right]$, ni para la interacción maniobra por orden de presentación $\left[F(10 / 36)=0.96, \quad M C_{c}=91.72, \quad p>.05\right]$, apareciendo tan sólo efectos significativos para las maniobras $([\mathrm{F}(2 / 36)=7.24$, $\left.\mathrm{MC}_{\mathrm{c}}=91.72, \mathrm{p}<.001\right]$. Realizado el mismo tipo de análisis con las puntuaciones diferenciales a la línea base inicial, tampoco aparecieron $\mathrm{ni}$ efectos del orden de presentación $[F(5 / 18)=0.43$, $M C_{c}=2$ I $\left.8.06, p>.05\right]$, ni efectos de interacción de maniobra por orden de presentación $\left[\mathrm{F}(10 / 36)=1.52, \quad \mathrm{MC}_{c}=37.37\right.$, p >.05], apareciendo de nuevo el efecto significativo para las maniobras $\left[\mathrm{F}(2 / 36)=13.99, \quad \mathrm{MC}_{c}=37.37, \mathrm{p}<.001\right]$. Las comprobaciones de pares de maniobras para ambos tipos de análisis dio de nuevo los mismos resultados en ambas, difiriendo significativamente la aritmética mental, tanto de la maniobra de Valsalva como de las contracciones musculares isométricas, no encontrándose diferencias significativas entre estas dos últimas.

Los análisis para las presiones arteriales presentaron los mismos únicos efectos para las maniobras, y los resultados de las comparaciones entre ellas son los mismos que se presentaron al principio de este apartado, por lo que no serán repetidos aquí. Un último y adicional análisis de los períodos de descanso o líneas base pretarea fue realizado en relación a la línea base inicial para los datos del TTP, no encontrándose en este caso ningún efecto significativo. Consiguientemente, la cuarta hipótesis no pudo confirmarse, ya que 
no se ha producido, al menos significativamente, ninguna caida en la reactividad de la presión, como lo demuestra la falta de significación del último análisis, así como la falta de efectos de orden o de interacción en los anteriores.

\section{DISCUSION}

Las correlaciones encontradas entre el TTP y la presión sistólica han sido altas y en la dirección esperada para toda la muestra de sujetos estudiada; éstos son totalmente acordes con los encontrados en un trabajo anterior (Fernández-Abascal, I983b); la diferencia que se presenta con otros trabajos en los que la mayor covariación se muestra con la presión arterial media (Obrist, Light, McCubbin, Hutcheson y Hoffer, I 979; Allen, Schneider, Davidson, Winchester y Taylor, 198I) puede ser explicada por la diferente forma de detección de la presión usada en los citados trabajos, que fue intra-arterial frente a la detección indirecta aquí empleada. Otro punto importante para la validación del TTP como índice de la presión es el de la influencia del PPE sobre el TTP; en este trabajo para la obtención de información acerca de este punto se incluyeron dos tareas experimentales, acerca de una de las cuales existe evidencia de que eleva la presión alterando el PPE (contracciones musculares isométricas), mientras que la otra la eleva sin la alteración (maniobras de Valsalva). Los resultados muestran que ambas tareas presentan un comportamiento distinto, lo cual pudiera tomarse como un indicio de que el PPE varía su aportación al TTP de unas tareas a otras, por lo que este punto deberá ser estudiado con una metodología más precisa, antes de utilizar el TTP como índice de la presión arterial.

Otra de las finalidades de este trabajo era el establecimiento de un índice de reactividad en presión arterial; en este caso la metodología empleada parece la adecuada, y la estandarización de una tarea estresante, por ejemplo la aritmética mental, que parece ser un buen indice de responsabilidad individual (Fredrikson, 1982), puede ser de gran utilidad terapéutica para la evaluación, tanto de la reactividad de la presión como de la influencia comportamental en la reactividad betaadrenérgica estimada por los cambios en el IEL, con este mismo procedimiento.

La explicación de por qué al comparar la tarea estresante con una línea base de relajación lleva a predicciones exitosas en reactividad de la presión arterial, y al hacerlo con referencia a una línea base pretarea se pierde esta capacidad, parece ser doble; por un lado, bajo relajación, la reactividad del sujeto está atenuada, obteniéndose así un rango mayor en variación, que evidencia más claramente a los sujetos más reactivos $\mathrm{y}$, por otro lado, la línea base pretarea es en alguna medida una evaluación "casual» o asituacional, puesto que al no tener el sujeto asignada ninguna tarea específica que realizar no se controla su actividad cognitiva, a la que es tan sensible la presión. Así, la obtención de una diferencia mínima entre la línea base pretarea y la tarea estresante no tiene por qué deberse a una baja reactividad en presión, sino que puede deberse a la tarea no controlada que esté realizando el sujeto durante la línea base; por contra, la obtención de un rango de variación amplio sí que será indicativo de alta reactividad, como hemos observado en algunos sujetos de este trabajo.

Por último, con respecto a la no aparición del efecto de caida de la presión arterial, a pesar de que parece ser un problema clásico, hay que señalar la existencia de dos diferencias importantes entre las circunstancias donde generalmente aparece y las que envuelvan nuestro trabajo; la primera de ellas es que el efecto de caída es un fenómeno típico de trabajos de feed-back, y éste no es nuestro caso, y en segundo lugar, que su aparición parece ser más marcada o crítica en tareas que conducen a una reducción en los niveles de presión, y éste tampoco es nuestro caso. Así pues, esta falta de aparición del fenómeno de caída puede estar debida precisamente a esas diferencias en 
el entorno general de la situación experimental y quizá principalmente a esas diferencias en las propias tareas experimentales, que, a su vez, influencian la propia situación experimental. Por otra parte, este resultado es equivalente al encontra- do recientemente por Bunell ( 1982 ), quien no encontró ni el efecto de caída ni diferencias entre el doble análisis; aunque en este caso también se emplearon tareas tendentes a producir aumentos en la presión arterial.

\section{Referencias}

Allien, R. A.; Schneider, J. A.; DAvidson, D. M.; Winchester, M. A., y TAYlor, C. B.: «The covariation of blood pressure and pulse transit time in hypertensive patients». Psychopbysiology, $301-306$. $19^{81}$.

Boyer, J. T.; Fraser, J. R. E., y Doyi.E, A. E.: "The hemodynamic effects of cold inmersion». Clinical Sciences, 19, 539-550. 1960 .

BUNNEL.I., D. E.: "Autonomic myocardial influences as a factor determining inter-task consistency of heart rate reactivity". Psychophysiology, 19, 442-448. 1982.

FERnindez-ABASCAL., E. G.: El tiempo de tránsito del pulso como indice de la presión arterial. Universidad Complutense de Madrid. Madrid, i $983 a$.

Fernandez-Abascai., E. G.: «Relación entre el tiempo de tránsito del pulso y la presión arterial». Informes de Psicologia, 2, $149-172$. $1983 \mathrm{~b}$.

FEY, S. G., y LiNDholm, E.: «Systolic blood pressure and heart rate changes during three sessions involving biofeedback or no feedback). Psychopbysiology, 12, 513-519. 1975 .

Fi.essas, A. P.; Kumar, S., y SPODick, D. H.: "Effects of valsalva maneouvre on the carotid systolic intervals: Beat to beat vs. time analysis. American Heart Journal, 80, 552-531. 1970.

FREDRIKSON, M.: "Essential hypertension and individual response specifity». Psychopbysiology, 19, 560 (abstract). 1982 .

GAVALAS, R. J.: "Operant reinforcement of an autonomic response: Two studies". Journal of the Experimental Analysis of Bebavior, 10, $119-130.1967$.

Harian, W. R.; Osborne, R. K., y Graybel, A.: «Prognostic values of the cold pressor test and the basal blood pressurem. American Journal of Cardiology, 13,683-687. 1964 .

HINES, E. A., y BROWN, G. E.: «The cold pressor test for measuring the reactivity of the blood pressure: Data concerning 571 normal and hypertensive subjects". American Heart Journal, 11, 1-9. 1936.

LIGHT, K. C., y OBRIST, P. A.: "Cardiovascular response to stress: Effects of opportunity to avoid shock experience and performance feedback". Psychopbysiology, I7, 243-292. 1980a.

LIGHT, K. C., y OBRIST, P. A.: "Cardiovascular reactivity to behavioral stress in young males with and without marginally elevated casual systolic pressures: A comparison of clinic, home and laboratory measures". Hypertension, 2, 802-808. $1980 \mathrm{~b}$.

MANUCK, S. B., y PRoietTI, J. M.: «Parental hypertension and cardiovascular response to cognitive and isometric challenge». Psychopbysiology, 19, 481-489. 1982.

NEWIIN, D. B., y LEVENSON, R. W.: «Pre-ejection period: Measuring beta-adrenergic influences upon the heartm. Psychophysiology, 16, 546-553. 1979.

Obrist, P. A.: Cardiovascular Psycbopbysiology. Plenum Press. Nueva York, 1981.

Obrist, P. A.; Light, K. C.; HASTRup, J. L., y LANGER, A. W.: "Beta-adrenergic hyperreactivity and behavioral stress: A precursor to established essential hypertension?" En S. M. WEAISS, J. A. HERD y B. H. FOX (Eds.): Perspectives on Behavioral Medicine. Academic Press. Nueva York, 1981 .

Obrist, P. A.; Light, K. C.; McCubbin, J. A.; Hutcheson, J. S., y Hoffer, J. L.: «Pulse trabsit time: Relationship to blood pressure and myocardial performancen. Psychophysiology, 16, 292-301. 1979.

QuARrY, V. M., y SPODICK, D. H.: "Cardiac responses to isometric exercise". Circulation, 49, 905-920. 1974.

Remington, R. D.; LAMbrath, B.; MOSER, M., y HoObler, S. W.: “Circulatory reactions of normotensives and hypertensives subjects and of the children of normal and hypertensiva parents". American Heart Journal, s9, \$8-70. 1960.

SHAPIRO, A. P.: «An experimental study of comparative responses of blood pressure to different noxious stimulin. Journal of Chronic Diseases, 13, 293-311. 1961.

ShapiRo, A. P.; MOUSTSOS, S. E., y KRIFCHER, E.: «Patterns of pressor response to noxious stimuli in normal, hypertensive and diabetic subjects». Journal of Clinical Investigation, 42, 1890-1898. 1963.

ShapiRo, D.; TURSKY, B., y SCHWARTZ, G. E.: "Control of blood pressure in man by operant conditioning. Supplement In, Circulation Research, 26, 127-132. 1970.

STEPTOE, A.: «Blood pressure control: A comparison of feedback and instructions using pulse wave velocity measurements". Psychopbysiology, 13, 528-535. 1976.

STEPTOE, A.: «Voluntary blood pressure reductions measured with pulse transit time: Training conditions and reactions to mental work». Psychophysiology, 14, 492-498. 1977. 


\section{Estudios}

STEPTOE, A.: «Control of cardiovascular reactivity and treatment of hypertension». En R. S. SURwiT, R. B. Williams, A. Steptoe, y R. Biersner (Eds.): Bebavioral Treatment of Disease. Plenum Press. Nueva York, 1982.

StePtoe, A.; Smulyan, H., y Gribiin, B.: «Pulse wave velocity and blood pressure change: Calibration and applicationsm. Psychopbysiology, $13,488-493.1976$.

\section{Resumen}

Los propósitos de este trabajo fueron: primero, evaluar el grado con el cual el tiempo de tránsito del pulso (TTP) covaria con la presión sistólica; segundo, estimar la influencia del periodo de preegección sobre el TTP; tercero, probar un indice de reactividad en la presión arterial, y cuarto, estudiar el decremento en la actividad cardiovascular a lo largo del tiempo.

Los resultados revelan una alta correlación entre el TTP y la presión sistólica $(\bar{r}=-.866)$, aunque el TTP probablemente no sólo refleje cambios de la presión arterial, sino también del periodo de preeyectión.

La respuesta ante una tarea de aritmética mental, en contraste con una linea base de relajación, se mostró como un buen indice de la reactividad en la presión sistólica.

El cuarto propósito no pudo realizarse por no producirse el decremento esperado en la actividad cardiovascular.

\section{Abstract}

The purpose of this research was: first to evaluate the degree to which pulse transit time (PTT) covaries with systolic blood pressure, second to estimate the influence of pre-ejection period on PTT, third to test an index of blood pressure reactivity and fourth to study the decrease in cardiovascular activity along a period of time.

The results show a bigh correlation between PTT and blood pressure $(\bar{r}=-.866)$, though PTT most probably shows changes not only in the blood pressure but also in the pre-ejection period.

The response on mental aritmetic task proved to be a good index of systolic blood pressure reactivity in contrast to a baseline of relaxation.

The fourth purpose was reached since the expected decrease in the cardiovascular activity did not take place. 